\title{
BMJ Open Impact of a multifactorial treatment programme on clinical outcomes and cardiovascular risk estimates: a retrospective cohort study from a specialised diabetes centre in Denmark
}

Narges Safai, ${ }^{1}$ Bendix Carstensen, ${ }^{2}$ Henrik Vestergaard, ${ }^{1,3}$ Martin Ridderstråle ${ }^{4,5}$

To cite: Safai N, Carstensen B, Vestergaard $\mathrm{H}$, et al. Impact of a multifactorial treatment programme on clinical outcomes and cardiovascular risk estimates: a retrospective cohort study from a specialised diabetes centre in Denmark. BMJ Open 2018:8:e019214. doi:10.1136/ bmjopen-2017-019214

- Prepublication history for this paper is available online. To view these files, please visit the journal online (http://dx.doi. org/10.1136/bmjopen-2017019214).

Received 17 August 2017 Revised 23 January 2018 Accepted 14 February 2018

Check for updates

${ }^{1}$ Patient Care, Steno Diabetes Center Copenhagen, Gentofte, Denmark

${ }^{2}$ Clinical Epidemiology, Steno Diabetes Center Copenhagen, Gentofte, Denmark

${ }^{3}$ Section of Metabolic Genetics, Novo Nordisk Foundation of Basic Metabolic Research, University of Copenhagen, Copenhagen, Denmark

${ }^{4}$ Clinical Pharmacology, Novo Nordisk A/S, Søborg, Denmark

${ }^{5}$ Department of Clinical

Sciences, Lund University, Malmö, Sweden

Correspondence to

Narges Safai;

narges.safai@regionh.dk

\section{ABSTRACT}

Objectives To investigate the impact of a multifactorial treatment programme in a real-life setting on clinical outcomes and estimated cardiovascular disease (CVD) risk.

Design A retrospective observational cohort study, using data from the electronic medical records and national registers.

Setting Tertiary diabetes centre in Denmark.

Participants Patients with type 2 diabetes $(n=4299)$ referred to a programme with focus on treatment of hyperglycaemia, hypertension and dyslipidaemia between 1 January 2001 and 1 April 2016.

Outcomes Primary outcomes were changes in haemoglobin $\mathrm{A} 1 \mathrm{c}\left(\mathrm{HbA}_{1 \mathrm{c}}\right)$, blood pressure (BP) and lowdensity lipoprotein (LDL) cholesterol as well as proportion reaching treatment targets. Our secondary outcome was to investigate changes in antidiabetic, antihypertensive and lipid-lowering treatment, together with the impact on estimated CVD risk. Linear mixed model for repeated measurements were used for continuous variables and logistic regression for dichotomous variables.

Results The patients achieved a mean $\pm S D$ decrease in $\mathrm{HbA}_{1 \mathrm{c}}$, systolic and diastolic $\mathrm{BP}$ and $\mathrm{LDL}$ cholesterol of $1.0 \% \pm 0.04 \%(10.6 \pm 0.4 \mathrm{mmol} / \mathrm{mol}), 6.3 \pm 0.4 \mathrm{~mm} \mathrm{Hg}$, $2.7 \pm 0.2 \mathrm{~mm} \mathrm{Hg}$ and $0.32 \pm 0.02 \mathrm{mmol} / \mathrm{L}$, respectively $(p<0.0001)$. The proportion of patients who met the treatment goal for $\mathrm{HbA}_{1 \mathrm{c}}(<7 \%(<53 \mathrm{mmol} / \mathrm{mol}))$ increased from $31 \%$ to $58 \%(p<0.0001)$; for $\mathrm{BP}(<130 / 80 \mathrm{~mm} \mathrm{Hg})$ from $24 \%$ to $34 \%(p<0.0001)$, and for LDL cholesterol $(<2.5 \mathrm{mmol} / \mathrm{L}$ (patients without previous CVD) or $<1.8 \mathrm{mmol} / \mathrm{L}$ (patients with previous CVD)) from $52 \%$ to $65 \%$. Those reaching all three guideline treatment targets increased from $4 \%$ to $15 \%(p<0.0001)$, and when relaxing the BP target to $<140 / 85$ from $8 \%$ to $24 \%$. The estimated CVD risk was relatively reduced by $15.2 \%$ using the Swedish National Diabetes Register risk engine and $30.9 \%$ using the UK Prospective Diabetes Study risk engine.

Conclusions Our data support that short-term multifactorial treatment of patients with glycaemic dysregulation in a specialist outpatient setting is both achievable and effective, and associated with a clinically meaningful improvement in CVD risk.
Strengths and limitations of this study

- Large cohort of dysregulated patients with type 2 diabetes under real-world conditions and strong validity of data with repeated recordings of clinical measurements and access to national registries.

- Selection bias in terms of more motivated and highrisk patients being referred to the clinic, and by exclusion of those who did not show up.

- The use of risk engines can only give an estimate of the cardiovascular disease risk and the UK Prospective Diabetes Study risk engine is based on a population many years prior to ours where treatment guidelines were different.

\section{INTRODUCTION}

Type 2 diabetes is an increasing global health threat. It is estimated that 439 million people will be diagnosed with diabetes by 2030. ${ }^{1}$ Type 2 diabetes is associated with an increased risk of microvascular complications such as nephropathy, neuropathy and retinopathy as well as macrovascular disease, resulting in a decreased life expectancy and substantial personal and societal expenses. ${ }^{2}$ Ensuring good glycaemic control remains the most effective therapeutic measure to reduce the risk of developing microvascular disease. ${ }^{34}$ Multifactorial treatment with tight control of glycaemia, blood pressure (BP) and lipids, accompanied by acetylsalicylic acid (ASA) and lifestyle advice, is known to reduce progression of microvascular complications, cardiovascular disease (CVD) and mortality by $50 \%$ in patients with type 2 diabetes and microalbuminuria. ${ }^{5-7}$ Consequently, diabetes guidelines have advocated an intensified treatment approach aiming at addressing and reducing all CVD risk factors in patients with diabetes since several years. ${ }^{89}$ 
For most patients, sufficient glycaemic, BP and lipid control can be achieved in a primary care setting but in high-risk patients, or in patients with complex treatment regimens, the proportion of patients who achieve metabolic control in primary care is lower. ${ }^{1011}$ In this situation, in most healthcare systems, high-risk patients are referred to specialist clinics for evaluation. A broad riskfactor intervention in this subgroup has proven particularly effective in the Steno-2 study. ${ }^{5}$ However, it remains unknown whether the results seen in the study setting can be achieved in clinical practice.

The overall aim of this study was to describe how the multifactorial intervention methods from the Steno-2 study perform in a larger scale clinical setting. Our primary objective was to describe changes in metabolic outcomes as a result of such structured short-term intervention and to test for gender differences. Our secondary objective was to describe the pharmacological changes and to evaluate the impact on estimated CVD risk by using two different risk assessment tools: the UK Prospective Diabetes Study (UKPDS) risk engine ${ }^{12}$ and the 5-year Swedish National Diabetes Registry (NDR) risk model. ${ }^{13}$

\section{METHODS}

\section{Design and setting}

This study is based on patients referred to Steno Diabetes Center (SDC), a tertiary multidisciplinary and highly specialised diabetes centre in the Capital Region of Denmark. It serves as one out of three referral centres with a catchment area of over 1.7 million people and provides diabetes care on a permanent basis to about 5600 patients. During the Steno-2 study, SDC designed a treatment programme algorithm specifically for patients with type 2 diabetes and glycaemic dysregulation. The primary goal of the programme is to improve patient quality of life and reduce mortality by prevention of acute and chronic complications of diabetes. This is done by motivating and encouraging self-management, professional support in behavioural changes, and pharmacological treatment according to national and international guidelines. The SDC Type 2 Clinic (T2C) opened in 2001, providing care for patients referred from general practitioners (GPs) or other hospitals in the region. Patients were referred to the clinic either as newly diagnosed with a need for education and start of treatment, requiring a shift to insulin treatment, having micro- or macrovascular complications, or having glycaemic dysregulation in spite of attempts to control the disease by the GP. The programme, which is still running and is the same for all patients, involves a consultation with a nurse, a dietitian and a physician in a structured order with specific assignments and is comparable to the intensive treatment arm of the Steno-2 study (figure 1). The individual visits are, depending on the need, complemented by optional group-based theme sessions with the overall aim of facilitating patient empowerment and with phone consultations from a nurse. The treatment programme consist

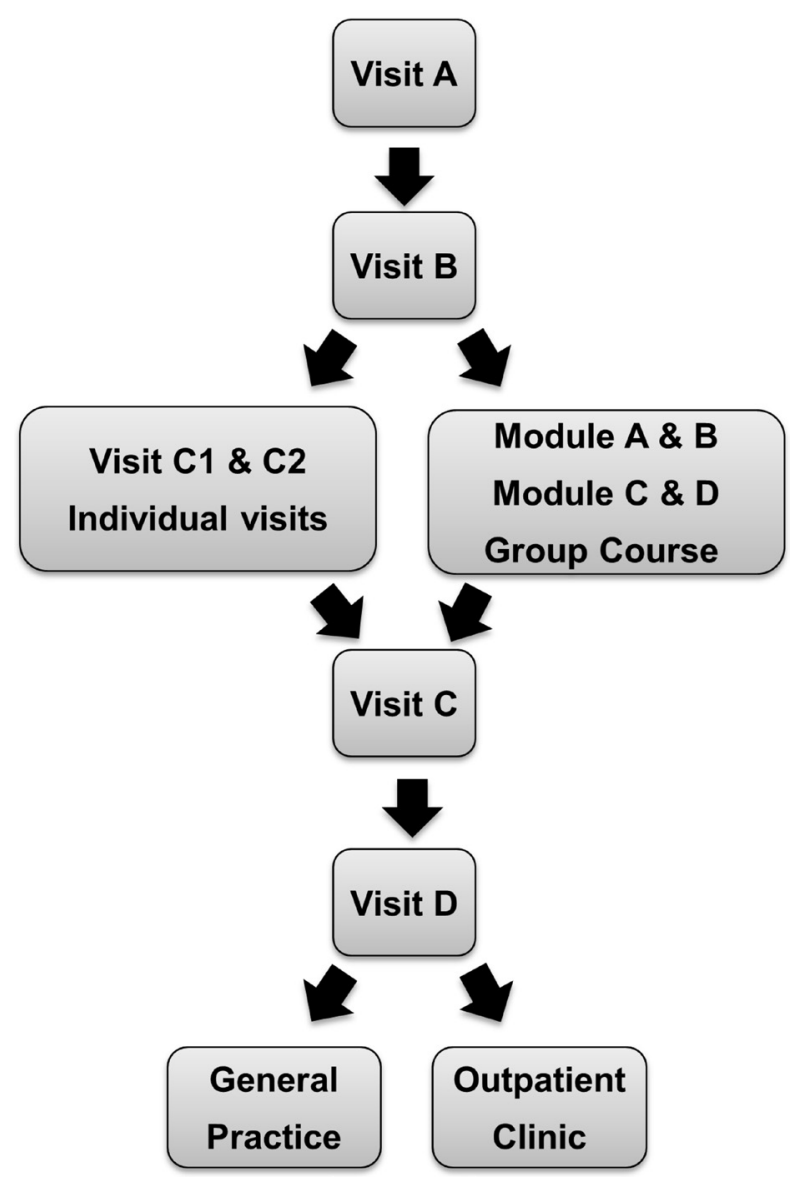

Figure 1 Flow chart of the treatment programme. Visit A: visit at the laboratory, eye clinic and consultation with nurse. Visit B: consultation with nurse, dietitian and physician. Visit C1 and C2: individual programme with nurse. Group sessions: module A: 'Me and my diabetes'; module B: 'My feet and physical activity'; module C: 'My diet'; module D: 'My motivation and future lifestyle plans'. Visit C: consultation with nurse and dietitian. Visit D: final visit with nurse, dietitian and endocrinologist. After approximately 8 months, patients with no complications are referred back to general practice and those with microvascular or macrovascular complications are referred to the outpatient clinic.

of self-management training with a focus on knowledge, lifestyle behaviour including diet, physical activity and smoking cessation, skills to improve glycaemic control such as self-monitoring of blood glucose and skills to prevent and identify complications. Furthermore, there is focus on pharmacological treatment of hyperglycaemia, hypertension and dyslipidaemia. After approximately 8 months, patients were evaluated for referral back to their GP, or to continue at the SDC outpatient clinic. The structure of programme has remained unchanged in the study period while, for example, medications used have followed updated treatment guidelines. The Danish treatment guidelines have followed the international guidelines from European Association for the Study of Diabetes (EASD) and American Diabetes Association (ADA) and were revised in 2003, 2011 and 2014. ${ }^{14-16}$ We defined the baseline and evaluation follow-up visits as the first and last 
visits to the $\mathrm{T} 2 \mathrm{C}$, respectively. This study is a retrospective observational study with demographics, clinical and laboratory information extracted from the electronic medical records and laboratory database of SDC.

\section{Study population}

We included all patients who had finalised a treatment programme between 1 January 2001 and 1 April 2016 $(\mathrm{n}=4489)$, and to avoid no-shows, once off or very brief consultations, we excluded patients with a treatment duration under 30 days (ie, between the baseline and follow-up visits, $n=190$ ). We ended up with a total of $\mathrm{n}=4299$ patients. Sixteen per cent of the patients were subsequently re-referred to the clinic, but we only included their first treatment programme here. All data were anonymised prior to analysis.

\section{Subject characteristics}

Laboratory analyses at the baseline visit were encouraged to be fasting and included: glucose, haemoglobin A1c $\left(\mathrm{HbA}_{1 \mathrm{c}}\right)$, haemoglobin $(\mathrm{Hb})$, creatinine, total cholesterol, high-density lipoprotein (HDL) cholesterol, low-density lipoprotein (LDL) cholesterol, triglycerides (TG), C-peptide and urine albumin. At all in-between visits and at follow-up, an $\mathrm{HbA}_{1 \mathrm{c}}$, $\mathrm{BP}$ and weight were measured. All laboratory and anthropometric measurements were recorded using standardised procedures at the SDC-accredited laboratory (ISO 15189). Body mass index (BMI) was calculated from weight and height $\left(\mathrm{kg} / \mathrm{m}^{2}\right)$. A person was considered overweight at BMI $\geq 25 \mathrm{~kg} / \mathrm{m}^{2}$ and obese at BMI $\geq 30 \mathrm{~kg} / \mathrm{m}^{2}$. For BP and heart rate, automated oscillometric BP recorders were used (AND UA-787plus, A\&D medical, California, USA). Smoking status was obtained at every visit.

\section{Diabetes complications and pharmacological treatment}

Microalbuminuria was here defined as a morning urine sample with urine albumin of $30-300 \mathrm{mg} / \mathrm{L}$ or urine albumin-to-creatinine ratio $>30 \mathrm{mg} / \mathrm{g}$ to $300 \mathrm{mg} / \mathrm{g}$ at the first visit. Macroalbuminuria likewise but with a value $>300 \mathrm{mg} / \mathrm{L}$ or $>300 \mathrm{mg} / \mathrm{g}$. Peripheral neuropathy was defined by examining vibration sensation with a biothesiometer and using an age-adjusted threshold. ${ }^{17}$ Information on CVD was obtained from The National Patient Register and included diagnosis from 1977 to 2015 and procedures from 1995 to 2015. Prior CVD was defined as one or more of the following: myocardial infarction, heart surgery, ischaemic heart disease, heart insufficiency, atrial fibrillation, vascular surgery, stroke, transitory cerebral ischaemia and amputations using International Classification of Diseases (ICD) 8 and ICD-10 codes.

Information on medication was obtained by Register of Medicinal Products Statistics, where individual-level data on all prescription drugs sold in Danish community pharmacies since 1994 have been recorded and administered by Statistics Denmark. ${ }^{18}$ A person was defined as being on a treatment at baseline if they had purchased a prescribed drug less than 180 days before their first visit and at follow-up if they purchased a prescribed drug after their first visit and less than 30 days after their last visit.

Permission to use data has been obtained from the Danish Data Protection Agency (ref. number: 2007-580015) and from the Danish Patient Safety Authority. According to Danish Committee, law register studies do not require an approval from the National Committee on Health Research Ethics.

\section{CVD risk}

To evaluate the effect of changes in metabolic outcomes on the estimated risk of CVD, we calculated CVD risk at baseline and at follow-up using two different risk assessment tools: a Swedish risk model specific for type 2 diabetes ${ }^{13}$ and the UKPDS risk engine. ${ }^{12}$ The Swedish model is based on patients with type 2 diabetes using 12 predictors: sex, age, diabetes duration, TG, HDL cholesterol, $\mathrm{HbA}_{1 \mathrm{c}}$, systolic $\mathrm{BP}$, BMI, smoking status, albuminuria, atrial fibrillation and previous CVD. It is derived from a large observational sample of patients $(\mathrm{n}=24288)$ in the Swedish NDR followed from 2002 to 2007 and estimates the 5 year risk of CVD. The UKPDS risk engine is also type 2 diabetes-specific and based on 4540 patients from the UKPDS trial (1977 to 1991). It includes $\mathrm{HbA}_{1 \mathrm{c}}$ as a continuous variable and calculates the risk of developing a new coronary heart disease (CHD) event.

\section{Statistical methods}

The primary outcomes were changes in blood glucose control $\left(\mathrm{HbA}_{1 \mathrm{c}}\right), \mathrm{BP}$ and lipids from first visit (baseline) to end of treatment (follow-up evaluation visit) and to explore gender differences in outcomes. Furthermore we investigated how many patients reached the recommended targets for $\mathrm{HbA}_{1 \mathrm{c}}$ (A), BP (B) and LDL cholesterol (C) according to national guidelines, ${ }^{14}$ collectively referred to as $\mathrm{ABC}$ control: $\mathrm{HbA}_{1 \mathrm{c}}<7 \%(<53 \mathrm{mmol} / \mathrm{mol})$, $\mathrm{BP}<130 / 80 \mathrm{~mm} \mathrm{Hg}$ and LDL cholesterol $<2.5 \mathrm{mmol} / \mathrm{L}$ $(<100 \mathrm{mg} / \mathrm{dL}$, patients without previous CVD) or $<1.8 \mathrm{mmol} / \mathrm{L} \quad(<70 \mathrm{mg} / \mathrm{dL}$, patients with previous CVD). For blood lipids, the T2C programme assumed they would not deteriorate if they were on target at baseline, and measurements were only repeated in case they were not at target at baseline. Accordingly, for this analysis a last observation carried forward approach was used to impute missing data. Student's t-test was used to test for gender differences at baseline or at follow-up. Comparison between baseline and follow-up was made using mixed model for repeated measurements (MMRM) for continuous variables adjusting for gender and baseline values and with the subject as a random effect. For dichotomous variables, for example, pharmacological treatment, logistic regression models were used adjusting for gender. McNemar test was used to compare changes in categorical variables. For risk estimates, exact 95\% CIs were calculated. SAS Enterprise Guide V.7.1 (SAS 
Institute) was used for database management and all of the above-mentioned analyses.

\section{RESULTS}

\section{Study cohort characteristics}

Baseline characteristics of the study cohort are shown in table 1 . The majority of patients were Caucasians, and $19 \%$ were diagnosed with diabetes within a year before their referral. There were more males $(n=2567)$ than females $(\mathrm{n}=1732)$ but no difference in treatment duration: median treatment programme duration was 8.4 months (IQR: 6.1, 11.3). There were more male smokers and ex-smokers. Males had a higher level of $\mathrm{HbA}_{1 c}$, BP, weight and TG but lower BMI and cholesterol levels at baseline (table 1).

\section{Metabolic outcomes}

There was a significant decrease in $\mathrm{HbA}_{1 \mathrm{c}}$ between baseline and follow-up of $1.0 \% \pm 0.04 \%(10.6 \pm 0.4 \mathrm{mmol} / \mathrm{mol})$, with males having a greater decrease than females even after adjusting for age and BMI $(\mathrm{p}=0.005)$. The decrease in systolic BP was $6.3 \pm 0.4 \mathrm{~mm} \mathrm{Hg}$ and in diastolic $\mathrm{BP}$ $2.7 \pm 0.2 \mathrm{~mm} \mathrm{Hg} \quad(p<0.0001$ for both $)$. The effect of treatment on BP was the same in both genders. There was a significant decrease in total-cholesterol, LDL and TG of $0.39 \pm 0.03 \mathrm{mmol} / \mathrm{L}, 0.32 \pm 0.02 \mathrm{mmol} / \mathrm{L}$ and $0.22 \pm 0.05 \mathrm{mmol} / \mathrm{L}$, respectively. There was no change in HDL levels overall $(p=0.2)$. As expected, females had higher HDL levels than males, both at baseline and at follow-up $(p<0.0001)$. This gender difference was also seen for total- and LDL-cholesterol levels where females had higher levels at both baseline and follow-up. The effect of treatment on lipid levels was equal in both genders.

\section{$\mathrm{ABC}$ control}

In general, the proportion of patients achieving full $\mathrm{ABC}$ control according to national guideline treatment targets increased from $4 \%$ to $15 \% \quad(p<0.0001)$. More females were achieving all three treatment targets at both baseline $(\mathrm{p}=0.047)$ and at follow-up $(\mathrm{p}=0.014)$. Patients achieving the $\mathrm{HbA}_{1 \mathrm{c}}$ target increased from $31 \%$ to $58 \%(\mathrm{p}<0.0001)$, the BP target from $24 \%$ to $34 \%$ ( $\mathrm{p}<0.0001)$, and the LDL target from $52 \%$ to $65 \%$ ( $\mathrm{p}=0.002$, figure 2 ). If the BP target was relaxed from $<130 / 80 \mathrm{~mm} \mathrm{Hg}$ to $<140 / 85 \mathrm{~mm}$ $\mathrm{Hg}$ the percentage achieving the BP target increased from $43 \%$ at baseline to $58 \%$ at follow-up $(\mathrm{p}<0.0001)$, and consequently full $\mathrm{ABC}$ control from $8 \%$ at baseline to $24 \%$ at follow-up $(\mathrm{p}<0.0001)$.

\section{Changes in pharmacological treatment}

The most common antidiabetic drug at baseline was metformin, which $58.4 \%$ of the patients were on, followed by sulphonylurea (SU), $38.4 \%$ and insulin, $19.5 \%$ (figure 3). Only a small proportion of patients were on dipeptidyl peptidase 4 (DPP-4) inhibitors, $7.0 \%$, glucagon-like peptide 1 (GLP-1) analogues, 3.9\% or other antidiabetic drug, $4.2 \%$. In general there was an increase in the use of medication during the programme. The largest increase was seen in use of metformin to $75.3 \%$, insulin to $36.9 \%$ and GLP1-analogues to $11.6 \%$. While SU only increased slightly to $41.8 \%$, DPP-4 inhibitors to $9.6 \%$ and other antidiabetics $4.3 \%$.

As part of the multifactorial treatment programme, we also observed an increase in use of antihypertensive drugs to $75.3 \%$, lipid lowering drugs to $75.9 \%$ and ASA to $69.6 \%$.

\section{Changes in cardiovascular risk}

Estimated baseline and follow-up cardiovascular risk according to the used risk engines are shown in table 2. Using the Swedish NDR model which predicts the 5 year risk of a new CVD event in a diabetic population, we observed a relative risk reduction of $15.2 \%$ (95\% CI: 14.5 to 15.9$)$. The UKPDS risk engine showed a relative risk reduction of $30.9 \%$ (95\% CI: 30.3 to 31.5 ) in the 5 year CHD risk estimate. Females had a lower risk than males both at baseline and at follow-up according to both risk models $(\mathrm{p}<0.0001)$. Meanwhile, both according to the Swedish NDR model and the UKPDS risk engine, females had a smaller relative risk reduction compared with males $(\mathrm{p}<0.0001)$.

\section{DISCUSSION}

This study shows that a short-term targeted multifactorial treatment programme in a specialised clinical setting can improve metabolic outcome measures and CVD risk in patients with type 2 diabetes and high prevalence of complications. This confirms that multifactorial treatment not only works in a clinical study setting, but is also feasible and effective in real world clinical practice. With a specialised group of healthcare providers and a structured treatment and educational programme that focuses on lifestyle intervention, self-management training and pharmacological treatment of hyperglycaemia, hypertension and dyslipidaemia, it is possible to accomplish significant CVD risk reductions in a high-risk population with diabetes.

\section{ABC control}

Intensive multifactorial intervention in high-risk patients has previously been shown to reduce CVD and mortality, ${ }^{7}$ and a recent 21 years' follow-up of the Steno-2 study population shows that patients in the intensive-therapy group survived for a median of 7.9 years longer than the conventional-therapy group patients. ${ }^{19}$ Here we show that the same treatment programme also works in clinical practice in a more diverse population, and results in a substantial reduction of 5- and 10-year CVD risk as estimated by two of the available and commonly used risk engines. In terms of risk factor intervention, glucose control continuous to be the greatest challenge to diabetes care. Nonetheless, all but $21 \%$ of patients changed from a higher to a lower $\mathrm{HbA}_{1 \mathrm{c}}$ category in this follow-up. Importantly, 
Table 1 Baseline characteristics of the study cohort

\begin{tabular}{|c|c|c|c|c|}
\hline & $\mathbf{N}$ & All & Females $(n=1732)$ & Males $(n=2567)$ \\
\hline Age (years) & 4299 & $59.3(12.4)$ & $59.9(12.9)$ & $58.9(12.1)$ \\
\hline Weight (kg) & 4256 & $91.5(21.2)$ & $84.4(20.4)$ & $96.3(20.4)$ \\
\hline Smokers, N (\%) & 4071 & $1629(37.9)$ & $622(35.9)$ & $1007(39.5)$ \\
\hline Caucasians, N (\%) & 4289 & $3724(87)$ & $1457(84)$ & $2267(89)$ \\
\hline Duration of type 2 diabetes (years) & 4252 & $7.1(6.5)$ & $7.3(6.6)$ & $6.9(6.5)$ \\
\hline Diabetes duration <1 year, N (\%) & 4252 & $828(19.5)$ & $311(18.1)$ & $517(20.4)$ \\
\hline GAD65 antibodies $\geq 25 \mathrm{U} / \mathrm{mL}, \mathrm{N}(\%)$ & 2376 & $116(2.7)$ & $59(3.4)$ & $57(2.2)$ \\
\hline $\mathrm{HbA}_{1 \mathrm{c}}(\%)$ & 4253 & $8.2(3.9)$ & $8.1(3.9)$ & $8.2(3.9)$ \\
\hline $\mathrm{HbA}_{1 \mathrm{c}}(\mathrm{mmol} / \mathrm{mol})$ & 4253 & $66(19)$ & $65(19)$ & $66(19)$ \\
\hline Prior cardiovascular disease, N (\%) & 4299 & $1127(26)$ & $400(23)$ & $727(28)$ \\
\hline Microalbuminuria, N (\%) & 4299 & $787(18)$ & $254(15)$ & $533(21)$ \\
\hline Macroalbuminuria, N (\%) & 4299 & $211(5)$ & $47(3)$ & $164(6)$ \\
\hline eGFR (mL/min) & 1335 & $78(17)$ & $77(18)$ & $79(16)$ \\
\hline Simple retinopathy, $\mathrm{N}(\%)$ & 3859 & $1134(29)$ & $422(27)$ & $712(31)$ \\
\hline Proliferative retinopathy, N (\%) & 3859 & $56(1)$ & $25(2)$ & $31(1)$ \\
\hline Peripheral neuropathy, N (\%) & 2343 & $549(23)$ & $140(15)$ & $409(29)$ \\
\hline \multicolumn{5}{|l|}{ Blood pressure } \\
\hline Systolic (mm Hg) & 4280 & $141.7(21.7)$ & $140.5(22.5)$ & $142.6(21.1)$ \\
\hline \multicolumn{5}{|l|}{ Medication } \\
\hline Metformin, N (\%) & 4299 & $2511(58)$ & $1025(59)$ & $1486(58)$ \\
\hline Sulfonylurea, N (\%) & 4299 & $1652(38)$ & $673(39)$ & $979(38)$ \\
\hline DPP-4 inhibitor, N (\%) & 4299 & $303(7)$ & $126(7)$ & $177(7)$ \\
\hline GLP-1 analogue, N (\%) & 4299 & $168(4)$ & $73(4)$ & $95(4)$ \\
\hline Insulin, N (\%) & 4299 & $836(19)$ & $346(20)$ & $490(19)$ \\
\hline Other OAD, N (\%) & 4299 & $179(4)$ & $67(4)$ & $112(4)$ \\
\hline RAS blockade, N (\%) & 4299 & $2027(47)$ & $736(42)$ & $1291(50)$ \\
\hline All antihypertensive drugs, N (\%) & 4299 & 2678 (62) & 1099 (63) & 1579 (62) \\
\hline Lipid lowering drug, N (\%) & 4299 & $1988(46)$ & $776(45)$ & $1212(47)$ \\
\hline Acetylsalicylic acid, N (\%) & 4299 & $1538(36)$ & $530(31)$ & 1008 (39) \\
\hline
\end{tabular}

Values are means (SDs) unless stated otherwise.

*Prior CVD was defined as one or more of the following: myocardial infarction, heart surgery, ischaemic heart disease, heart insufficiency, vascular surgery, stroke, transitory cerebral ischaemia, amputation.

CVD, cardiovascular disease; DPP-4, dipeptidyl peptidase 4; eGFR, estimated glomerular filtration rate; GAD, glutamic acid decarboxylase; GLP-1, glucagon-like peptide $1 ; \mathrm{HbA}_{1 \mathrm{c}}$, haemoglobin $\mathrm{A}_{1 \mathrm{c}}$; $\mathrm{HDL}$, high-density lipoprotein; LDL, low-density lipoprotein; OAD, oral antidiabetic drug; RAS, renin-angiotensin system. 


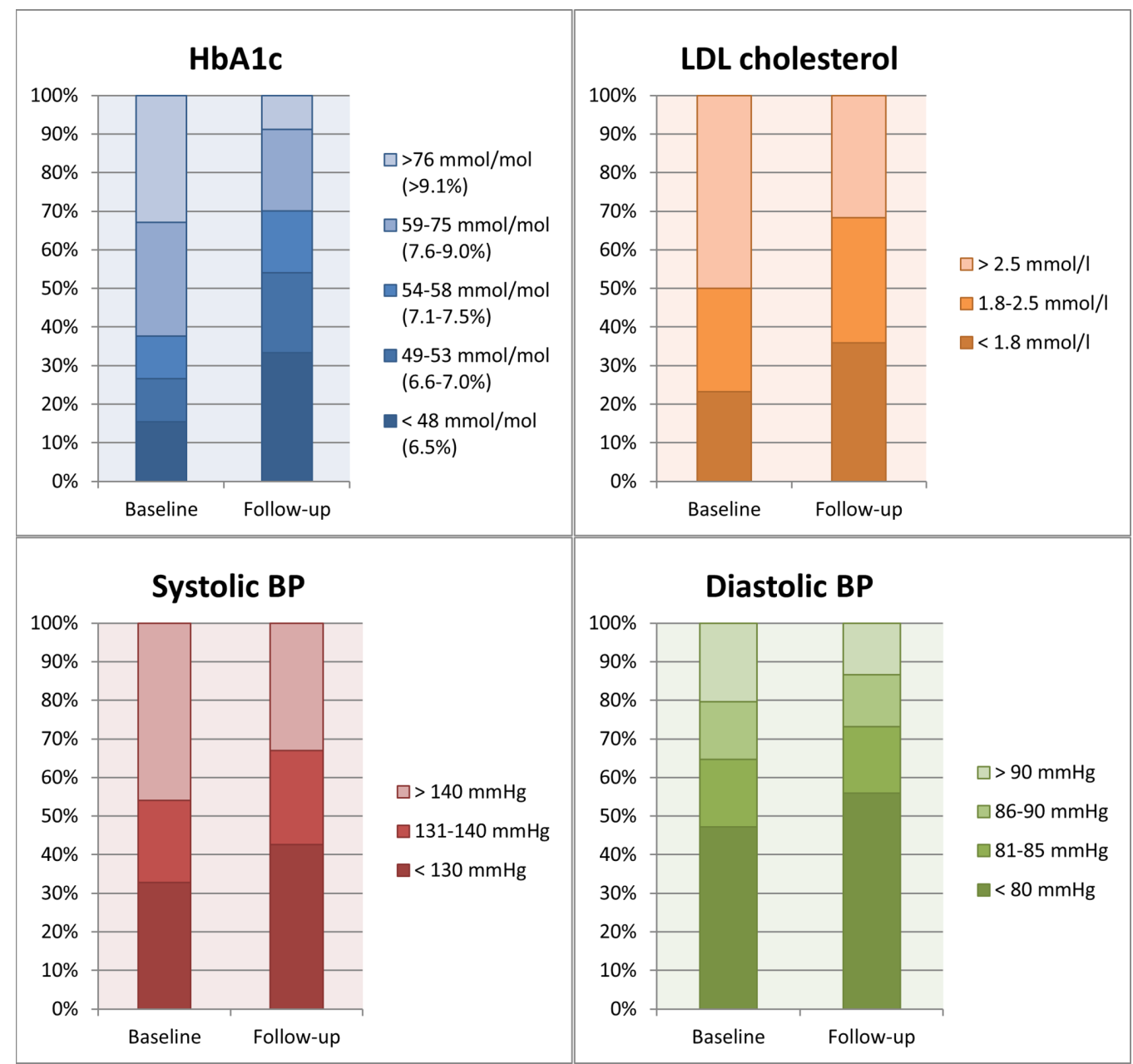

Figure 2 Proportion of patients achieving the treatment targets for haemoglobin $\mathrm{A}_{1 \mathrm{c}}$, low-density lipoprotein (LDL) cholesterol, systolic and diastolic blood pressure (BP) at baseline and at follow-up.

the improvement in glycaemic control was not accompanied by a general increase in weight. In fact, although we found that $15 \%$ of those in the normal weight category shifted to the overweight category when comparing the changes in BMI categories, $15 \%$ of those who were in the obese or overweight category dropped to a lower weight category. The weight gain observed in some patients is probably explained by the increased use of insulin, while weight loss in others can be explained by an increased use of GLP-1 receptor agonist treatment in recent years along with lifestyle management including dietary and physical activity advice.

With focus on hyperglycaemia, hypertension and dyslipidaemia, we found an increase in the proportion of patients achieving the recommended targets that are comparable to intervention studies. ${ }^{20}{ }^{21}$ Here, the relative proportion of patients achieving $\mathrm{HbA}_{1 c}<53$ $\mathrm{mmol} / \mathrm{mol}(7 \%)$ nearly doubled, $\mathrm{BP}<130 / 80 \mathrm{~mm} \mathrm{Hg}$ increased by $42 \%$ and $\mathrm{LDL}<2.5 \mathrm{mmol} / \mathrm{L}$ by $25 \%$. The T2C treatment programme in this complex high-risk cohort resulted in a higher prevalence of risk factors in control equal to what has been observed in the more general diabetes population in the National Health and Nutrition Examination Surveys (NHANES) from
2007 to $2010 .^{22}$ The NHANES data differ in the way that their data were cross-sectional with participants with self-reported diabetes, without any distinction between type 1 and type 2, and with a different risk profile. Our population was more selected by being referred from their GP and requiring specialised care, which means they either had more comorbidities or a more complex treatment than the general patient with type 2 diabetes. For $\mathrm{HbA}_{1 \mathrm{c}} 58 \%$ in our cohort achieved the treatment target vs $53 \%$ in the NHANES cohort and for LDL-cholesterol $65 \%$ vs $56 \%$, respectively. But for BP there was a big difference, $34 \%$ in our cohort vs $51 \%$ in the NHANES cohort. This could be due to a higher prevalence of high BP in this group of patients selected with complex disease and long diabetes duration. We did observe a time trend in the data as the proportion of patients achieving the stringent BP target increased from 23\% in 2001 to $44 \%$ in 2015. The same improvement trend over time was observed in the proportion of patients achieving all three ABC targets; $7 \%$ in 2001 increasing to an average of $16 \%$ from 2006 and forward in our material and in the NHANES data from $7 \%$ in $1999-2002$ to $19 \%$ in 2007-2010. 


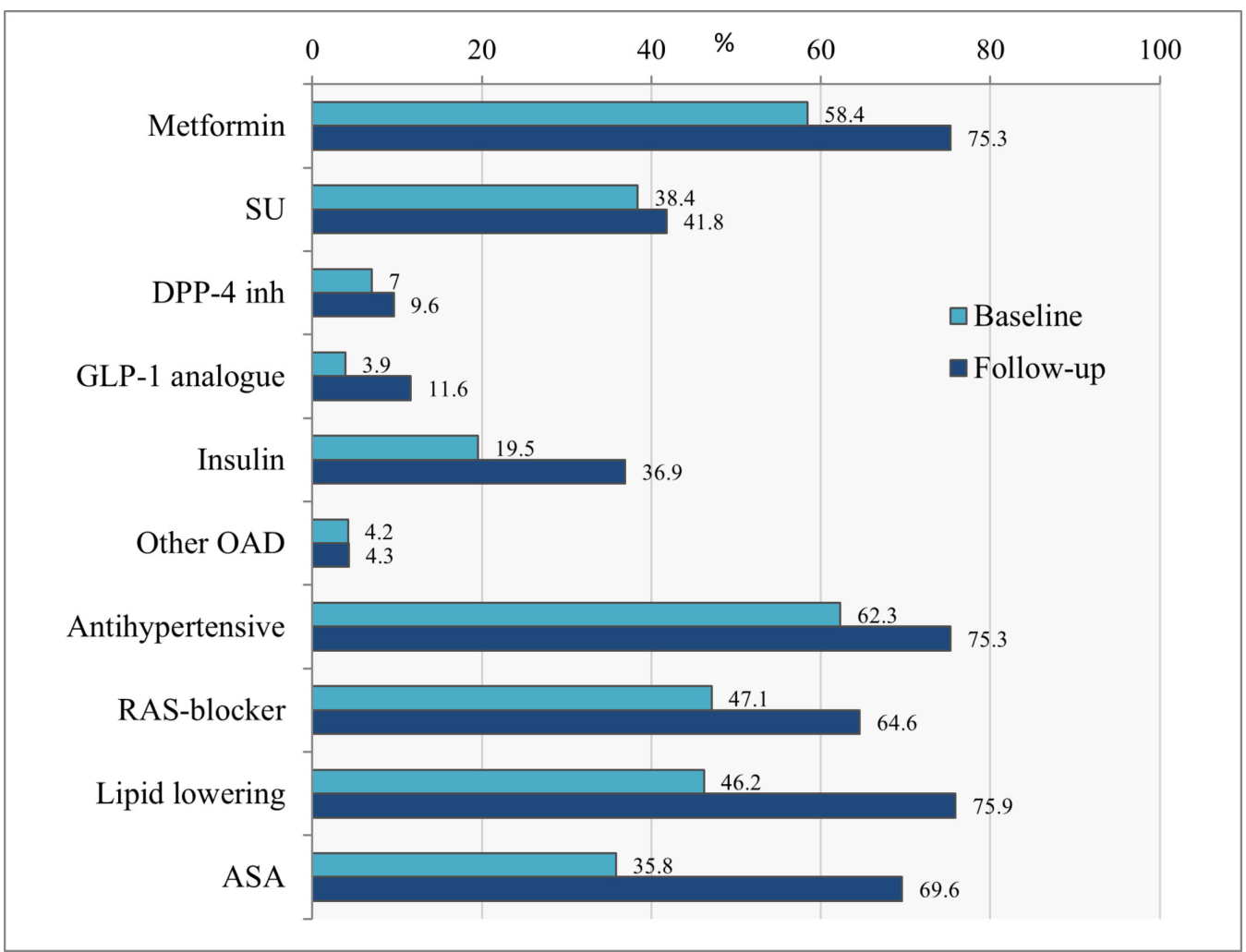

Figure 3 Proportion of patients on pharmacological treatment at baseline and follow-up. ASA, acetylsalicylic acid; DPP4, dipeptidyl peptidase 4; GLP, glucagon-like peptide; OAD, oral antidiabetic drug; RAS, renin-angiotensin system; SU, sulphonylurea.

\section{CVD risk}

To estimate the CVD risk in patients with type 2 diabetes is helpful to follow-up on treatment and to target further

\begin{tabular}{|c|c|c|}
\hline & Baseline & Follow-up \\
\hline \multicolumn{3}{|c|}{ Estimated CVD 5-year risk: NDR risk engine: } \\
\hline All & $29.8(19.6-44.6) \dagger$ & $25.0(16.6-37.4) \ddagger^{*}$ \\
\hline $\mathrm{F}$ & $24.9(15.9-37.0)$ & $21.1(13.7-31.1)^{\star}$ \\
\hline M & $34.0(22.6-48.2)$ & $28.1(19.1-41.2)^{\star}$ \\
\hline \multicolumn{3}{|c|}{ Estimated CHD 5-year risk: UKPDS risk engine: } \\
\hline All & $7.4(3.9-13.7) \S$ & $5.0(2.7-9.2) \rrbracket^{*}$ \\
\hline $\mathrm{F}$ & $4.8(2.6-8.7)$ & $3.3(1.9-5.9)^{\star}$ \\
\hline M & $9.6(5.3-16.7)$ & $6.4(3.7-11.3)^{\star}$ \\
\hline \multicolumn{3}{|c|}{ Estimated CHD 10-year risk: UKPDS risk engine: } \\
\hline All & $17.1(9.3-30.4) \S$ & $11.8(6.5-21.1) \rrbracket^{*}$ \\
\hline $\mathrm{F}$ & $11.4(6.1-20.0)$ & $7.9(4.5-14.0)^{*}$ \\
\hline M & $22.1(12.6-36.2)$ & $15.0(9.0-25.4)^{\star}$ \\
\hline
\end{tabular}

Estimated CVD risk according to the Swedish NDR risk engine and the estimated CHD risk according to the UKPDS risk engine. Data are median risk in \% (IQR).

${ }^{*} \mathrm{P}<0.0001$.

$\dagger n=3865 ; \ddagger n=3730 ; \S n=3895 ; \emptyset n=3757$.

$\mathrm{CHD}$, coronary heart disease; CVD, cardiovascular disease; $\mathrm{F}$, female, M, male, NDR, National Diabetes Registry; UKPDS, UK Prospective Diabetes Study. measures to patients at risk. In this study we used two different CVD risk engines to estimate the effect of the treatment programme. The UKPDS risk engine is diabetes-specific and has several advantages as it incorporates $\mathrm{HbA}_{1 \mathrm{c}}$ and diabetes duration as continuous variables. ${ }^{12}$ However, it is still not ideal as it is based on the patients recruited by UKPDS for randomisation in a clinical trial two decades ago before newer and more effective treatments were available or widely used (eg, statins, ACE inhibitors and antidiabetic drugs). Accordingly, recent validation showed poor calibration and overestimation of the CHD risk. ${ }^{23}$ A model that seems more suitable for our population is the Swedish NDR risk model, which is based on a more recent and nationwide population, reflecting a more diverse population and taking into account the history of previous CVD and BMI. By using this model we found a relative reduction in the estimated 5 year CVD risk of $16 \%$, after approximately 8 months, despite the increase in age and diabetes duration. Notably, the fact that $26 \%$ of the patients had a prior CVD diagnosis at baseline reflects the high risk profile and complexity of the population that was referred to the treatment programme. This of course does not normalise their actual risk, which will still be high, but can be a motivating factor for the patients that there are some modifiable risk factors that can reduce their risk. In comparison, the population used in the Swedish model had a mean risk of $11.9 \%$ 
and a 5 year risk of fatal/non-fatal CVD of more than $10 \%$ is defined as high risk. According to this $92 \%$ of our population was in high risk at baseline. The UKPDS risk engine which estimates the risk of the first CHD event in 5 or 10 years, gives a lower 5 - and 10-year risk estimate at baseline than the Swedish model, $7.4 \%$ and $17.1 \%$ respectively. This is nearly the same as the 10 year risk found in a NHANES population from 2007 to 2012 of $16.5 \%$ if no risk factors were in control and $10.2 \%$ if all risk factors were in control when using the same risk engine. $^{24}$

Interestingly, both the NDR and UKPDS risk engines estimated a higher risk reduction in males than in females. This, perhaps expected finding, could be due to the higher CVD risk in males at baseline, but could also be due to a relatively greater $\mathrm{HbA}_{1 \mathrm{c}}$ reduction seen in males, which is used in both the NDR and the UKPDS risk engines. However, the gender difference remained significant after excluding $\mathrm{HbA}_{1 \mathrm{c}}$ from the equation. This in spite a higher percentage of females achieved the metabolic targets.

\section{Strengths and limitations}

Strengths of this study include the validity of data with repeated recordings of the $\mathrm{HbA}_{1 \mathrm{c}}$ and $\mathrm{BP}$ at each visit, and that it includes a large cohort of patients treated under real-life conditions such that results might have greater external validity than the highly selected populations in randomised controlled trials (RCTs). Still, we cannot rule out that there might be a selection bias in terms of more motivated patients being referred to the clinic, and by exclusion of those who did not show up.

As a result of using a database and a register, we do not have complete data on all patients, and therefore, the cohort size changes a bit as results are based on those without missing values. Another limitation is that there is a lack of patient reported outcomes, such as adverse events of drugs and general well-being. This was not possible to extract from the electronic medical records. Furthermore we cannot be sure that the patients going through a treatment programme actually completed the programme or was discharged for other reasons. It is also important to acknowledge that most of the treatment programmes analysed here were completed before many of the new anti-diabetic treatments, as GLP1-analogues and sodium-glucose co-transporter 2 (SGLT2)-inhibitors, were widely used and before acceptance of a more personalised treatment as recommended in the position statement from ADA and EASD in 2012. ${ }^{16}$ Another limitation of this study is the use of risk engines that only give an estimate of the CVD or CHD risk and that UKPDS is based on a population many years prior to ours and treatment guidelines were not the same.

Meanwhile, the data serve as a baseline benchmark for real world data studies as to what can be achieved in routine clinical practice before these treatments and guidelines get wider use and implementation.
The use of a more individualised treatment approach with involvement of the patient in decision making is increasingly used in the treatment programmes at the moment and is expected to increase adherence to therapy. Furthermore, the combination of a more individualised $\mathrm{HbA}_{1 c}$ target and a broader selection of antidiabetic, antihypertensive and dyslipidaemia treatment will likely increase the proportion of patients achieving their treatment goals and thereby reduce their CVD risk and mortality. Specifically, since the EMPA-REG OUTCOME trial showed a $38 \%$ and the LEADER trial a $22 \%$ relative risk reduction in deaths from CVD events in patients with type 2 diabetes and a high risk of cardiovascular events, ${ }^{25}{ }^{26}$ this gives us further treatment options in this patient group. Therefore constant evaluation of the effects of our treatments on the risk of CVD or mortality is necessary. Combining these drugs, the treatment programme evaluated here, and an individualised approach would be a logical next step for future studies.

\section{CONCLUSION}

This study of patients with type 2 diabetes who undergo structured treatment programme lasting less than 1 year show that it is possible to increase the proportion of patients achieving the target levels for $\mathrm{HbA}_{1 c}, \mathrm{BP}$ and LDL, thereby reducing their estimated CVD and CHD risk. To the strengths of such a structured programme, we count the focus on treatment targets by a multidisciplinary team and the fact that it is time limited, which reduces clinical inertia and costs. Our results show that intensive treatment is not only effective in the RCT setting, but also in clinical practice and should encourage other healthcare systems to establish similar programmes.

Contributors NS and BC were responsible for data management and statistical analysis. NS and MR were responsible for interpretation of data and writing of the article. HV was responsible for interpretation and critical revision of the article. All authors fully approved the final version of the article.

Funding This study has been funded by Innovation Fund Denmark.

Competing interests NS, BC and HV were employed at Steno Diabetes Center A/S, now known as Steno Diabetes Center Copenhagen, and MR was employed there when the study was initiated. Steno Diabetes Center A/S was a research hospital working in the Danish National Health Service and owned by Novo Nordisk A/S. NS and $B C$ own shares in Novo Nordisk A/S.

Patient consent Not required.

Provenance and peer review Not commissioned; externally peer reviewed.

Data sharing statement Clinical data will be available at request, but information on diagnosis and medication is not allowed to be shared by the Danish National Patient Register or Statistics Denmark.

Open Access This is an Open Access article distributed in accordance with the Creative Commons Attribution Non Commercial (CC BY-NC 4.0) license, which permits others to distribute, remix, adapt, build upon this work non-commercially, and license their derivative works on different terms, provided the original work is properly cited and the use is non-commercial. See: http://creativecommons.org/ licenses/by-nc/4.0/

(C) Article author(s) (or their employer(s) unless otherwise stated in the text of the article) 2018. All rights reserved. No commercial use is permitted unless otherwise expressly granted. 


\section{REFERENCES}

1. Guariguata L, Whiting DR, Hambleton I, et al. Global estimates of diabetes prevalence for 2013 and projections for 2035. Diabetes Res Clin Pract 2014;103:137-49.

2. Sarwar N, Gao P, Seshasai SR, et al. Diabetes mellitus, fasting blood glucose concentration, and risk of vascular disease: a collaborative meta-analysis of 102 prospective studies. Lancet 2010;375:2215-22.

3. Holman RR, Paul SK, Bethel MA, et al. 10-year follow-up of intensive glucose control in type 2 diabetes. N Engl J Med 2008;359:1577-89.

4. Anon. Effect of intensive blood-glucose control with metformin on complications in overweight patients with type 2 diabetes (UKPDS 34). Lancet 1998;352:854-65.

5. Gaede P, Vedel P, Larsen N, et al. Multifactorial intervention and cardiovascular disease in patients with type 2 diabetes. $N$ Engl $J$ Med 2003;348:383-93.

6. UKPDS. Intensive blood-glucose control with sulphonylureas or insulin compared with conventional treatment and risk of complications in patients with type 2 diabetes (UKPDS 33). UK Prospective Diabetes Study (UKPDS) Group. Lancet 1998;352:837-53.

7. Gaede $\mathrm{P}$, Lund-Andersen $\mathrm{H}$, Parving $\mathrm{HH}$, et al. Effect of a multifactorial intervention on mortality in type 2 diabetes. $N$ Engl J Med 2008;358:580-91.

8. IDF Clinical Guidelines Task Force. Global guideline for Type 2 diabetes. Brussels: International Diabetes Federation, 2005.

9. Rydén L, Grant PJ, Anker SD, et al. ESC Guidelines on diabetes, prediabetes, and cardiovascular diseases developed in collaboration with the EASD: the Task Force on diabetes, pre-diabetes, and cardiovascular diseases of the European Society of Cardiology (ESC) and developed in collaboration with the European Association for the Study of Diabetes (EASD). Eur Heart J 2013;34:3035-87.

10. Kotseva K, Wood D, De Backer G, et al. EUROASPIRE III. Management of cardiovascular risk factors in asymptomatic high-risk patients in general practice: cross-sectional survey in 12 European countries. Eur J Cardiovasc Prev Rehabil 2010;17:530-40.

11. Alonso-Fernández M, Mancera-Romero J, Mediavilla-Bravo JJ, et al. Glycemic control and use of A1c in primary care patients with type 2 diabetes mellitus. Prim Care Diabetes 2015;9:385-91.

12. Stevens RJ, Kothari V, Adler Al, et al. United Kingdom Prospective Diabetes Study G. The UKPDS risk engine: a model for the risk of coronary heart disease in Type II diabetes (UKPDS 56). Clin Sci 2001;101:671-9.

13. Zethelius B, Eliasson B, Eeg-Olofsson $\mathrm{K}$, et al. A new model for 5 -year risk of cardiovascular disease in type 2 diabetes, from the Swedish National Diabetes Register (NDR). Diabetes Res Clin Pract 2011;93:276-84.
14. Sundhedsstyrelsen CfEoMT. Type 2-diabetes. Medicinsk teknologivurdering af screening, diagnostik og behandling. $2003 \mathrm{htt}$ ps://www.sst.dk/ /media/F42943CEAEC743DDAD8CB57FF55C95 81. ashx

15. Snorgaard ODT, Breum L. Farmakologisk behandling af type 2-diabetes - mål og algoritmer - 2014. 2014 http://www.endocrin ology.dk/PDF/FarmakologiskbehandlingDM2rev2014.pdf

16. Inzucchi SE, Bergenstal RM, Buse JB, et al. Management of hyperglycaemia in type 2 diabetes: a patient-centered approach Position statement of the American Diabetes Association (ADA) and the European Association for the Study of Diabetes (EASD). Diabetologia 2012;55:1577-96.

17. Bloom S, Till S, Sönksen P, et al. Use of a biothesiometer to measure individual vibration thresholds and their variation in 519 non-diabetic subjects. Br Med J 1984;288:1793-5.

18. Kildemoes HW, Sørensen HT, Hallas J. The Danish National Prescription Registry. Scand J Public Health 2011;39(7 Suppl):38-41.

19. Gæde P, Oellgaard J, Carstensen B, et al. Years of life gained by multifactorial intervention in patients with type 2 diabetes mellitus and microalbuminuria: 21 years follow-up on the Steno-2 randomised trial. Diabetologia 2016;59:2298-307.

20. Anon. Tight blood pressure control and risk of macrovascular and microvascular complications in type 2 diabetes: UKPDS 38. UK Prospective Diabetes Study Group. BMJ 1998;317:703-13.

21. Griffin SJ, Borch-Johnsen K, Davies MJ, et al. Effect of early intensive multifactorial therapy on 5-year cardiovascular outcomes in individuals with type 2 diabetes detected by screening (ADDITION-Europe): a cluster-randomised trial. Lancet 2011;378:156-67.

22. Stark Casagrande S, Fradkin JE, Saydah $\mathrm{SH}$, et al. The prevalence of meeting A1C, blood pressure, and LDL goals among people with diabetes, 1988-2010. Diabetes Care 2013;36:2271-9.

23. Bannister CA, Poole CD, Jenkins-Jones S, et al. External validation of the UKPDS risk engine in incident type 2 diabetes: a need for new type 2 diabetes-specific risk equations. Diabetes Care 2014;37:537-45

24. Wong ND, Patao C, Malik S, et al. Preventable coronary heart disease events from control of cardiovascular risk factors in US adults with diabetes (projections from utilizing the UKPDS risk engine). Am J Cardiol 2014;113:1356-61.

25. Zinman B, Wanner C, Lachin JM, et al. Empagliflozin, Cardiovascular Outcomes, and Mortality in Type 2 Diabetes. N Engl J Med 2015;373:2117-28.

26. Marso SP, Daniels GH, Brown-Frandsen K, et al. Liraglutide and Cardiovascular Outcomes in Type 2 Diabetes. N Engl J Med 2016;375:311-22. 